\title{
DETERMINANTES DO CONSUMO DE TABACO DURANTE A GRAVIDEZ E VIGILÂNCIA DE GRAVIDEZ
}

\author{
Emília Coutinho \\ Professor in Superior Health School of Viseu \\ Polytechnic Institute of Viseu - PhD \\ ecoutinhoessv@gmail.com \\ Carlos Pereira \\ Professor in Superior Health School of Viseu \\ Polytechnic Institute of Viseu - PhD \\ Paula Nelas \\ Professor in Superior Health School of Viseu \\ Polytechnic Institute of Viseu - PhD \\ Cláudia Chaves \\ Professor in Superior Health School of Viseu \\ Polytechnic Institute of Viseu - PhD \\ Odete Amaral \\ Professor in Superior Health School of Viseu \\ Polytechnic Institute of Viseu - PhD \\ Carla Cruz \\ Professor in Superior Health School of Viseu \\ Polytechnic Institute of Viseu - PhD
}

Fecha de Recepción: 4 Marzo 2018

Fecha de Admisión: 10 Abril 2018

\section{RESUMO}

Enquadramento: Uma gravidez sem consumo de tabaco traduz-se em mais saúde para a mãe e para a criança. A vigilância de gravidez assegura a identificação atempada de fatores de risco e a possibilidade de uma assistência pré-natal precoce que previna complicações. 0 enfermeiro assume-se como um profissional de charneira na promoção de saúde e prevenção de complicações no acompanhamento da mulher/casal ao longo da gravidez e na consciencialização da importância de adoção de estilos de vida saudáveis.

Objetivo: Identificar os determinantes do consumo de tabaco e vigilância da gravidez.

Metodologia: Trata-se de um estudo quantitativo, transversal, descritivo e correlacional, com uma amostra de 3232 mulheres que foram mães. 0 estudo foi realizado em 32 maternidades/hospitais com maternidade, de Norte a Sul de Portugal, no serviço de obstetrícia/internamento de puér- 
peras, entre os anos de 2010 e 2012. 0 instrumento de colheita de dados foi o questionário que permitiu a caracterização sociodemográfica da amostra com uma idade média das mulheres de 28,75 anos ( $d p= \pm 5,463$ anos), $78,2 \%$ de nacionalidade portuguesa e $21,8 \%$ estrangeiras. Foram assegurados os procedimentos éticos, e o estudo teve a autorização da Comissão Nacional de Proteção de Dados para além dos pareceres favoráveis das Comissões de ética das instituições envolvidas. Os dados foram tratados com o programa SPSS (Statistical Package for the Social Sciences) versão 24 para Windows.

Resultados: A mulher que vive com companheiro, e que não planeou a gravidez tem maior probabilidade de vigilância adequada de gravidez e não consumir tabaco durante a gravidez. Por outro lado, o local de residência em meio rural, mulher com formação profissional superior ou formação técnica, que não exerce uma profissão, que teve problemas de saúde durante a gravidez e que não efetuou preparação para o parto apresenta menor probabilidade de não vigiar a gravidez e consumir tabaco.

Conclusão: A vigilância adequada de gravidez assegura a identificação atempada de fatores de risco como o consumo de tabaco e a possibilidade de uma sensibilização atempada da mulher/casal para uma gravidez sem tabaco e para a prevenção de complicações.

Palavras-chave: determinantes; consumo; tabaco; gravidez; vigilância

\section{ABSTRACT}

Determinants of tobacco use during pregnancy and pregnancy surveillance.

Background: A non-smoking pregnancy translates into more health for the mother and the child. Pregnancy surveillance ensures the timely identification of risk factors and the possibility of early prenatal care that prevents complications. The nurse assumes himself as a professional in promoting health and preventing complications in the follow-up of the woman / couple throughout pregnancy and in the awareness of the importance of adopting healthy lifestyles.

Objective: Identify the determinants of tobacco use and pregnancy surveillance.

Methodology: This is a quantitative, cross-sectional, descriptive and correlational study with a sample of 3232 mothers. The study was carried out in 32 maternity / maternity hospitals, from north to south of Portugal, in the obstetrics / inpatient postpartum service between 2010 and 2012 . The data collection instrument was the questionnaire that allowed the sociodemographic characterization of the sample with a mean age of women of 28.75 years ( $S D=5.463$ years), $78.2 \%$ Portuguese and $21.8 \%$ foreign. Ethical procedures were ensured and the study was authorized by the National Data Protection Commission in addition to the favorable opinions of the ethics committees of the institutions involved. The data was processed with the SPSS program (Statistical Package for the Social Sciences) version 24 for Windows.

Results: A woman who lives with a partner who has not planned pregnancy is more likely to have adequate pregnancy surveillance and not consume tobacco during pregnancy. On the other hand, a woman living in a rural area, with a higher vocational training or technical training, who is not employed, has had health problems during pregnancy and has not been prepared for childbirth, is less likely to surveillance her pregnancy and more likely to be a tobacco user.

Conclusion: Adequate pregnancy surveillance ensures the timely identification of risk factors such as tobacco use and the possibility of timely sensitization of the woman / couple to a non-smoking pregnancy and for the prevention of complications.

Keywords: determinants; consumption; tobacco; pregnancy; surveillance 


\section{INTRODUÇÃO}

0 consumo de tabaco é, segundo a Organização Mundial de Saúde (World Health Organization, 2013) e a Direção-Geral da Saúde (2015, p. 10), a principal causa evitável de complicações na gravidez, para a mãe e feto/recém-nascido, estimando que 5,0\% a 8,0\% dos partos de pré-termo, $13,0 \%$ a $19,0 \%$ dos partos de crianças com baixo peso ao nascer, $5,0 \%$ a $7,0 \%$ das mortes associadas a partos de pré-termo e $23 \%$ a $34 \%$ das mortes súbitas do lactente, pudessem estar relacionadas com o consumo de tabaco durante a gravidez.

Tendo em conta "a composição química do tabaco, rico em nicotina, substância psicoativa geradora de dependência, e em substâncias cancerígenas, tóxicas e mutagénicas, não existem consumos mínimos seguros de exposição ao tabaco, o que exige a adoção de medidas preventivas e de proteção Direção-Geral da Saúde (2013, p. 1) pelo que devem ser criadas condições para que as mulheres grávidas usufruam do direito que têm a um ambiente livre de fumo em casa, no trabalho e em locais públicos (World Health Organization, 2013, p. 38; Portugal, Despacho 404, 2012)

André, Cândido, Santiago, Cruz, Carreira e Amendoeira (2015, p. 117) realizaram uma revisão sistemática da literatura com o objetivo de identificarem instrumentos para a caracterização do consumo de tabaco na mulher grávida. Da análise dos artigos, os autores verificaram que o consumo de tabaco durante a gravidez se revelava tendencialmente mais frequente quando associado às variáveis sociodemográficas, como o baixo nível de escolaridade e/ou elevados níveis de escolaridade, baixos níveis socioeconómicos e coabitação com fumadores. Os estudos analisados evidenciam que existe uma associação entre os sintomas depressivos pré-natais e/ou durante a gravidez e a dependência da nicotina. As mulheres com hábitos tabágicos e etílicos apresentam scores mais elevados de sintomas depressivos. André et al. (2015, p. 117) referem que, apesar de se dar enfoque à avaliação de determinadas dimensões da saúde mental, os estudos não se assemelham quanto às variáveis estudadas, o que não permite ter uma visão mais específica acerca das variáveis que melhor caraterizam o consumo de tabaco durante a gravidez. Ainda em conformidade com André et al. (2015, p. 115), no que se refere ao período pré-natal, há a preocupação em relacionar os hábitos tabágicos com outras variáveis, designadamente: "fatores psicossociais (humor pré-natal, sintomas depressivos e a violência contra a mulher grávida), sociodemográficos (idade gestacional e coabitantes), fatores ambientais (exposição ao fumo ambiental), fatores culturais e consumo de outras substâncias (álcool e substâncias ilícitas) ".

Em consonância com a Direção-geral de Saúde (2015, p. 11), o baixo nível de literacia é um fator preditor de maior probabilidade de consumo de tabaco e de exposição ao fumo durante a gravidez e preditor de menor probabilidade de sucesso na cessação tabágica. Vários autores (Reid et al., 2010; World Health Organization, 2010; Greaves, Poole, Okoli, 2011; Smedberg et al., 2014, cit. por Direcção-Geral da Saúde, 2015, p. 11) referem que a comorbilidade psiquiátrica, o consumo de álcool ou outras dependências, o facto de ser mãe solteira ou estar desempregada, são fatores que também estão associados aos consumos mais elevados de tabaco durante a gravidez. Deste modo, os profissionais de saúde, neste caso concreto os enfermeiros, têm de estar atentos às adolescentes grávidas, mães solteiras e/ou oriundas de meios menos favorecidos, social e economicamente, uma vez que estas podem necessitar de um apoio acrescido na cessação tabágica.

Em termos de implementação de estratégias de prevenção que autorizem aumentar o potencial de bem-estar materno-fetal, André et al. (2015, p. 114) referem que o maior desafio consiste na redução e/ou cessação do consumo de tabaco na mulher grávida. É que consumir tabaco durante a gravidez ou o facto de a mulher estar exposta de forma continuada a ambientes de fumo, ou seja, ser fumadora passiva, compromete gravemente a saúde da mãe e do feto. "A alta prevalência de fumar durante a gravidez, associada a uma diminuição da perfusão placentária contribui para 0 
aumento de abortos espontâneos, prematuridade, restrição de crescimento fetal e aumento das taxas de morbilidade e mortalidade perinatal e infantil" (André et al., 2015, p. 114). Na mesma linha, Miller, Cao, Wen, Yang, Lafleche e Walker, (2010, p. 334) referem que o consumo de tabaco durante a gravidez resulta em stresse oxidativo, diminui a concentração de oxigénio no sangue materno, compromete a maturação placentária, a oxigenação fetal, assumindo-se como fator de risco para a redução da função pulmonar nos primeiros anos de vida, asma, obesidade na criança e síndrome de morte súbita infantil.

De acordo com Miller et al. (2010, p. 334), o tabagismo materno e a pré-eclâmpsia aumentam de forma independente o risco de complicações pré e pós-natais. As grávidas com pré-eclâmpsia e que são fumadoras manifestam um risco superior de desenvolver complicações ao longo da gravidez comparativamente a grávidas com pré-eclâmpsia que não fumam. Del Bono, Ermisch e Francesconi (2012) fazem referência ao estudo de Marco Francesconi et al. (2012) com 17,483 puérperas, tendo o mesmo revelado que o consumo de tabaco durante a gravidez reduz 0 peso ao nascer em 140,0-160,0 gramas em recém-nascidos de mães americanas e 190,0 gramas em recémnascidos de mães britânicas, reduzindo igualmente 0 crescimento fetal em cerca de 4,0 gramas por semana nas grávidas de ambos os países. De acordo com a Organização Mundial de Saúde (2013), a exposição ao fumo do tabaco afeta todos os estádios da reprodução humana. 0 tabagismo afeta tanto a fecundidade masculina, como a feminina. 0 tabagismo materno está associado ao aumento dos riscos de gravidez ectópica, rotura prematura de membranas, desprendimento de placenta, placenta prévia, aborto espontâneo, morte fetal, parto prematuro, baixo peso ao nascer, recém-nascido pequeno para idade gestacional e anomalias congénitas, como a fissura labial. Após o nascimento, o risco de síndrome da morte infantil aumenta entre os bebés de mulheres que fumaram durante ou após a gravidez. Os danos causados pelo tabagismo na gravidez não se limitam apenas aos produtos do tabaco fumado. 0 consumo de tabaco na gravidez também está associado ao aumento do risco de baixo peso ao nascer (<2500 gramas ) em $22 \%$ dos casos. 0 tabagismo determina ainda hipoxia fetal pré-placentária pela diminuição da $\mathrm{pO}_{2}$ da mãe, além de causar vasoconstrição das artérias do espaço interviloso da placenta com consequente menor absorção de nutrientes"(Galão et al., 2009, p.219). De acordo com os mesmos autores, esse dano poderá repercutir-se na absorção de nutrientes com consequente "diminuição do ganho de peso fetal intrauterino". Sustentado em outros estudos, Galão et al. (2009, p. 219) referem que as placentas de mulheres com hábitos tabágicos durante a gravidez poderá igualmente revelar um aumento da calcificação, associando-se a "recém-nascidos abaixo do percentil 10 de crescimento com consequente alteração de morbidade e mortalidade neonatais", sendo esta uma evidência também referida por Correia, Nascimento, Gouveia, Martins, Sandes et al. (2007, p. 201), segundo os quais, o consumo de tabaco durante "a gravidez associa-se não só a acontecimentos perinatais nefastos, mas também a importantes repercussões pós-natais". Pelas consequências altamente prejudiciais do consumo de tabaco durante a gravidez traçou-se como objetivo identificar os determinantes do consumo de tabaco e vigilância da gravidez

\section{METODOLOGIA}

Trata-se de um estudo quantitativo, transversal, descritivo e correlacional, com uma amostra de 3232 mulheres que foram mães com uma idade média de 28,75 anos ( $d p= \pm 5,463$ anos). A colhei-ta de dados ocorreu em 32 maternidades/hospitais com maternidade, de Norte a Sul de Portugal, no serviço de obstetrícia/internamento de puérperas, entre os anos de 2010 e 2012 . Os critérios de inclusão considerados na realização do estudo foram:"(i) residir em Portugal há pelo menos 7 meses; (ii) ter realizado a vigilância do trabalho de parto, parto e puerpério numa maternidade por- 
tuguesa; (iii) ter pelo menos 24 horas de internamento após o parto; (iv) aceitar participar no estudo"

0 instrumento de colheita de dados foi 0 questionário que permitiu a caracterização sociodemográfica da amostra com 78,2\% das mulheres de nacionalidade portuguesa e 21,8\% estrangeiras. Para quantificar o consumo de tabaco durante a gravidez considerou-se não haver consumo de tabaco quando a grávida não fumou ativamente nenhum cigarro durante a gravidez, considerou-se consumo de tabaco durante a gravidez quando a grávida fumou ativamente durante a gravidez, independentemente da quantidade e da frequência. Apesar dos efeitos altamente nefastos do consumo passivo de tabaco o mesmo não foi tido em conta neste estudo.

As prevalências foram expressas em proporções e comparadas pelo teste do qui-quadrado, com aplicação de um nível de significância de 0,05. Determinou-se ainda a magnitude de associação entre as variáveis com recurso aos Odds Ratio (OR) e respetivos intervalos de confiança a 95\% (IC95\%).

Foram assegurados os procedimentos éticos, e o estudo teve a autorização da Comissão Nacional de Proteção de Dados para além dos pareceres favoráveis das Comissões de ética das instituições envolvidas. Os dados foram tratados com o apoio do programa SPSS (Statistical Package for the Social Sciences) versão 24 para Windows.

\section{RESULTADOS}

Para a determinação das variáveis preditoras do consumo de tabaco realizámos uma regressão logística binária com a qual se procurou avaliar a relação entre variáveis de contexto sociodemográfico (idade, estado civil, nacionalidade, habilitações literárias, local de residência, formação profissional, e profissão atual), variáveis obstétricas (número de gestações, número de filhos, idade gestacional e idade atual do filho), e contextuais à gravidez (planeamento da gravidez, internamentos durante a gravidez, problemas de saúde prévios, problemas de saúde durante a gravidez, presença do enfermeiro na consulta de vigilância da grávida e preparação para o parto) com o consumo de tabaco e vigilância durante a gravidez. De entre os métodos de estimação logística disponíveis para a seleção das variáveis optamos pelo método Forward Stepwise LR, porque neste método a entrada de uma variável independente no modelo é feita em função da significância estatística e a remoção de uma variável no modelo é feita a partir da significância do teste de rácio de verosimilhança, baseada nas estimações parciais da máxima verosimilhança do modelo.

0 número de participantes que estiveram incluídas no processo de análise foram 3144 a que corresponde $97.3 \%$ da totalidade da amostra tendo-se registado $2.7 \%$ de casos omissos. Foi atribuído 0 código 0 às mulheres com vigilância adequada da gravidez e sem consumo de tabaco durante a gravidez e com o código 1 às que não vigiaram a gravidez e que consumiam tabaco durante a gravidez. Foram executados oito passos para determinação das variáveis preditoras. A estimativa de probabilidade de sucesso registada no último passo, isto é, a probabilidade de as mulheres com vigilância adequada da gravidez e de não terem consumido tabaco é de $73,2 \%$ com um teste de qui quadrado Wald de $(X 2 w=623,617 ; p=0,000)$, revelando, assim, que pelo menos uma das variáveis independentes está relacionada com o modelo de regressão (logit $\pi$ ).

A sensibilidade do modelo é de $94,1 \%$, isto é, o modelo classifica corretamente as mulheres que vigiaram adequadamente a gravidez e não fumaram e a especificidade é de $23,6 \%$, ou seja, o modelo classifica corretamente as mulheres não vigiaram adequadamente a gravidez e que fumaram, com uma percentagem de casos corretamente classificados de $75,2 \%$.

Na tabela 1, em baixo, são apresentadas as variáveis preditoras e respetivos coeficientes e a sua significância. A coluna Exp (b) estima a probabilidade (odds rácio ajustado) da variável dependente por unidade da variável independente e a coluna \% $\operatorname{Exp(b)}$ as percentagens correspondentes. 
A mulher que vive com companheiro $(0 R=1,908)$, e que não planeou a gravidez $(O R=2.128)$ tem uma probabilidade aumentada em $90,8 \%$ e $112,8 \%$ respetivamente de vigiarem adequadamente a gravidez e não consumirem tabaco durante a gravidez.

Por outro lado, o local de residência em meio rural $(0 R=0,645)$, as mulheres com formação profissional superior ( $O R=0.537)$ ou com formação técnica $(O R=0,586)$, as que não exercem uma profissão ( $O R=0.640)$, as que tiveram problemas de saúde durante a gravidez $(O R=0.816)$ e as que não efetuaram preparação para o parto $(0 \mathrm{R}=0,570)$ a possibilidade de não vigiarem a gravidez e consumirem tabaco diminui em $35,5 \%, 46,3 \% 41,4 \% 36,0 \%, 18,4 \%$, e $43,0 \%$, respetivamente.

Tabela 10 - Coeficientes Logit do modelo de regressão logística da variável consumo de tabaco na gravidez com as variáveis preditoras

\begin{tabular}{|c|c|c|c|c|c|c|c|c|c|}
\hline Variáveis & b & s.e. & $\mathbf{X}^{2}$ Wald & gl & $\mathbf{p}$ & $\begin{array}{c}\operatorname{Exp}(B) \\
\text { OR } \\
\text { ajustado }\end{array}$ & $\% \operatorname{Exp}(B)$ & \multicolumn{2}{|c|}{$\begin{array}{l}\text { IC } 95 \% \\
\operatorname{Exp}(B)\end{array}$} \\
\hline Estado civil & 0,646 & 0,090 & 51,823 & 1 & 0,000 & 1,908 & 90,8 & 1,600 & 2,275 \\
\hline Local de residência & 0,439 & 0,119 & 13,731 & 1 & 0,000 & 0,645 & $-35,5$ & 0,511 & 0,813 \\
\hline $\begin{array}{l}\text { Formação profissional } \\
\text { (1) }\end{array}$ & $\begin{array}{c}- \\
0,622 \\
\end{array}$ & 0,100 & 38,541 & 1 & 0,000 & 0,537 & $-46,3$ & 0,441 & 0,653 \\
\hline $\begin{array}{l}\text { Formação profissional } \\
\text { (2) }\end{array}$ & $\begin{array}{c}- \\
0,534 \\
\end{array}$ & 0,132 & 16,470 & 1 & 0,000 & 0,586 & $-41,4$ & 0,453 & 0,759 \\
\hline Profissão atual (1) & 0,446 & 0,090 & 24,583 & 1 & 0,000 & 0,640 & -36 & 0,536 & 0,763 \\
\hline $\begin{array}{l}\text { Planeamento da } \\
\text { gravidez }\end{array}$ & $\begin{array}{c}- \\
0,755 \\
\end{array}$ & 0,089 & 72,516 & 1 & 0,000 & 2,128 & 112,8 & 1,788 & 2,532 \\
\hline $\begin{array}{l}\text { Problemas de saúde } \\
\text { durante a gravidez }\end{array}$ & $\begin{array}{c}- \\
0,204 \\
\end{array}$ & 0,094 & 4,699 & 1 & 0,030 & 0,816 & $-18,4$ & 0,678 & 0,981 \\
\hline
\end{tabular}

\section{DISCUSSÃO}

0 consumo de tabaco, por si só, tem muitas repercussões negativas sobre o organismo e esses efeitos podem ser maiores quando associados à gravidez. Fumar na gravidez é um fator responsável pelo aumento de casos de recém-nascidos com baixo peso no nascimento, partos prematuros e mortes perinatais (Machado \& Lopes, 2009, pp.75-76).0s mesmos autores documentam que há estudos que associam o tabagismo na gravidez com "alterações no desenvolvimento do sistema nervoso central e com a síndrome da morte súbita” (p. 76). A mulher que fuma na gravidez expõe 0 seu feto aos componentes do fumo do cigarro que atravessam a placenta e às modificações na oxigenação e metabolismo placentário, bem como às transformações no seu próprio metabolismo, secundárias ao fumo (Machado \& Lopes, 2009, p.76).

Aferiu-se que 0 estado civil, o local de residência, a formação profissional, a profissão, o planeamento da gravidez, os problemas de saúde durante a gravidez, e a preparação para o parto foram preditores do consumo de tabaco durante a gravidez.

No que se refere ao estado civil, verificou-se que $66,3 \%$ das mulheres com companheiro tiveram vigilância pré-natal e não consumo de tabaco durante a gravidez, mas é significativa a percentagem das que também possuem companheiro mas não tiveram vigilância na gravidez e com consumo de tabaco (46,7\%). 0 estudo de Motta, Echer e Lucena (2010) mostra que as mulheres mais propensas a parar de fumar antes da gestação foram as que 0 companheiro não fumava $(78,1 \%)$. Entre as que cessaram o hábito tabágico durante a gestação, 58,1\% tinham companheiros fumadores $(p=0,007)$. No grupo de mulheres grávidas a consumir tabaco, a percentagem de companheiros igualmente consumidores é de $36,7 \%$, e 0 das mulheres grávidas em abstinência atinge 37,3\%. 
De acordo com os resultados obtidos, no que se refere ao local de residência, apurou-se que nas mulheres não fumadoras $80,1 \%$ residem em meio urbano e 19,9\% em meio rural. Já por parte das mulheres fumadoras, $85,6 \%$ vivem em meio urbano e $14,4 \%$ em meio rural. Não se encontrando estudos que justifiquem estes resultados, poder-se-á dizer que o facto de 85,6\% das mulheres residentes em meio urbano terem consumido tabaco na gravidez poderá estar associado ao facto de no meio urbano ser mais "liberalizado" no seu consumo, ou seja, as mulheres que residem em meio rural poderão coibir-se mais desta prática, uma vez que se trata de meios mais pequenos e onde o fumar, por si só, ainda é uma prática pouco aceitável nas mulheres, com mais propensão à reprovação por parte de terceiros quando se trata de uma gravidez. Por outro lado, poderá justificar-se esta evidência com o pressuposto que no meio urbano o meio é mais influenciador/potenciador desta prática ou menos fiscalizador.

Fazendo-se uma análise dos resultados ao consumo de tabaco durante a gravidez em função da formação profissional, verifica-se que nas mulheres não fumadoras $43,5 \%$ não possuem formação, enquanto $41,3 \%$ têm formação superior. Nas mulheres fumadoras, a percentagem mais elevada corresponde às que não possuem formação profissional (63,8\%), secundadas pelas $24,7 \%$ das que possuem formação superior. Motta, Echer e Lucena (2010), na sua investigação, constataram que entre as mulheres grávidas que fumavam, 52,9\% tinha apenas 0 ensino básico, 55,9\% que fumavam estavam em abstinência e 47,3\% das que não fumavam possuíam 0 3.. ciclo do ensino básico. No grupo de mulheres que tinham o ensino superior, a maior percentagem é de não fumadoras $(11,5 \%)$.

\section{CONCLUSÕES}

Do estudo realizado, concluiu-se que há uma probabilidade de $72,3 \%$ de mulheres a efetuarem vigilância adequada da gravidez e de não terem fumado durante a mesma, enquanto 27,7\% não tiveram uma vigilância pré-natal adequada e fumaram durante a gravidez.

Concluiu-se que tanto fatores sociodemográficos como fatores contextuais à gravidez se constituem como preditores do consumo de tabaco durante a gravidez sendo que no decorrer das consultas de vigilância de gravidez se assegura a identificação atempada de fatores de risco como 0 consumo de tabaco, havendo a possibilidade de uma sensibilização atempada da mulher/casal para uma gravidez sem tabaco e para a prevenção de complicações.

Sugere-se a realização de outros estudos que procurem perceber quais os motivos que levam as grávidas à não cessação tabágica durante a gravidez, de modo a poder-se implementar intervenções no que diz respeito a esta problemática, informando as mulheres acerca dos riscos do consumo de tabaco durante a gravidez, disponibilizando-se intervenções psicossociais para a cessação tabágica às mulheres grávidas fumadoras ou que se encontrem em abstinência

\section{REFERÊNCIAS BIBLIOGRÁFICAS}

André, M., Cândido, A., Santiago, C., Cruz, O., Carreira, T., \& Amendoeira, A. (2015). Consumo de tabaco na mulher grávida: revisão sistemática da literatura. Revista Portuguesa de Enfermagem de Saúde Mental, Especial 2, 113-118. Acedido em http://www.scielo.mec.pt /pdf/rpesm/nspe2/nspe2a19.pdf

Correia, S., Nascimento, C., Gouveia, R., Martins, S., Sandes, A.R. et al. (2007). Gravidez e tabagismo uma oportunidade para mudar comportamentos. Acta Med Port, 20, 201-207. Acedido em https://www.actamedicaportuguesa.com/revista/index.php/amp/article/viewFile/858/532

Del Bono, Ermisch, J., \& Francesconi, M. (2012). Intrafamily resources allocations: A dynamic structural model of birth weight. Journal of Labour Economics, 30(4), 657-706.

Direcção-Geral de Saúde (2013). Programa Nacional para a Prevenção e Controlo do Tabagismo 
2012-2016. Programa Nacional para a Prevenção e Controlo do Tabagismo (PNPCT). DirecçãoGeral da Saúde. Lisboa. Acedido em http://www.sppneumologia.pt/uploads/files/comissoestrabalho/ct\%20tabagismo/tabagismo4.pdf

Direcção-Geral de Saúde (2015). Cessação tabágica na gravidez. Guia para profissionais de saúde. Programa Nacional para a Prevenção e Controlo do Tabagismo 2015. Direção-Geral da Saúde. Lisboa. https://www.dgs.pt/respire-bem1/documentos-e-publicacoes/cessacao-tabagica-nagravidez-guia-para-profissionais-de-saude.aspx

Galão, A.O., Sode, S.A., Gerhardt, M., Faertes, T.H., Krüger, M.S., Pereira, D.G., \& Borba, C.M. (2009). Efeitos do fumo materno durante a gestação e complicações perinatais. Rev HCPA; 29(3), 218-224.

Machado, J.B., Lopes, M.H.I. (2009). Abordagem do tabagismo na gestação. Scientia Medica, Porto Alegre, v. 19, 2, 75-80. Acedido em http://revistaseletronicas.pucrs.br/ojs/index.php/scientiamedica/article/viewFile/4718/7813

Miller, E.C., Cao, H., Wen, S.W., Yang. Q., Lafleche. J., \& Walker, M. (2010). The risk of adverse pregnancy outcomes is increased in preeclamptic women who smoke compared with nonpreeclamptic women who do not smoke. Am J Obstet Gynecol; 203(4), 334-338.

Motta, G.C.P., Echer, I.C., \& Lucena, A.F. (2010). Fatores associados ao tabagismo na gestação. Rev. Latino-Am. Enfermagem, 18(4), 1-8.

Portugal, Despacho n.ำ 404/2012 - Diário da República, 2. a série, n.ำ10, 13 de janeiro de 2012.

World Health Organization (2013). Recommendations for the prevention and management of tobacco use and second-hand smoke exposure in pregnancy. World Health Organization. Acedido em http://apps.who.int/iris/bitstream/10665/94555/1/9789241506076_eng.pdf 